Ann. Zootech., I970, 19 (2), 223-228.

\title{
DESCRIPTION D'UNE MACHINE A TRAIRE LES LAPINES
}

\author{
F. LEBAS \\ avec la collaboration technique de G. SAkpi et Y. LE Coz \\ Station de Recherches sur l'Élevage des Porcs, \\ Centre national de Recherches zootechniques, 78 -Jouy-en-Josas \\ Institut national de la Recherche agronomique
}

\begin{abstract}
RÉSUMÉ
Il est intéressant, à des fins de recherches, de connaître le potentiel laitier des lapines. La traite manuelle des lapines, même avec injection d'ocytocine, ne permet d'obtenir qu'une faible partic du lait présent dans la mamelle. Dans cet article est décrit un dispositif qui permet la traite mécanique de cette espèce. Le principe adopté est celui des trayeuses mécaniques pour Vache. La Lapine est suspendue dans un harnais au-dessus du système de récolte qui comprend Io gobelets trayeurs et un seul récipient de réception du lait. La vitesse de pulsation est de 45 coups par minute et la dépression varie entre 30 et $50 \mathrm{~cm}$ de mercure en fonction de l'étanchéité. Chaque gobelet trayeur est constitué d'un corps métallique, d'un manchon de caoutchouc. L'étanchéité avec la tuyauterie de réception est assurée par une simple gaine de caoutchouc. L'utilisation de cette machine permet de récolter des quantités de lait équivalentes à celles ingérées par les lapereaux en une tétéc, voire même plus importantes.
\end{abstract}

\section{INTRODUCTION}

Les divers auteurs ayant cherché à déterminer la composition du lait de lapine (Berguann et Turner, I937 ; Nixon, I953 ; NeUMeister et Krause, I956 ; Coates et al., I964; DAVIES et al., I964; CoWIE, I969) ont toujours pratiqué une traite manuelle qui permet d'obtenir des échantillons de Io à $30 \mathrm{~g}$ alors que les quantités delait présentes dans la mamelle sont de Ioo à $300 \mathrm{~g}$. NEUMEISTER et KRAUSE I956 avaient tenté une traite mécanique mais leurs efforts étaient restés infructueux. Le présent article a pour objet de décrire une machine à traire les lapines permettant un prélèvement de lait au moins égal aux quantités prélevées par les lapereaux. 


\section{I. - DESCRIPTION DE LA MACHINE A TRAIRE}

Sur un chariot mobile ont été réunis le système de contention de la lapine, une pompe à vide et la machine à traire proprement dite. Le principe de fonctionnement: de cette dernière est celui des appareils de traite mécanique des vaches laitières.

\section{A. - La machine à traire}

Entraînée par une pompe à palettes d'un débit nominal de $2 \mathrm{~m}^{3} / \mathrm{h}$, la machine est constituée par un pulsateur pneumatique, ro gobelets trayeurs et un dispositif de récolte du lait commun à l'ensemble (fig. I).

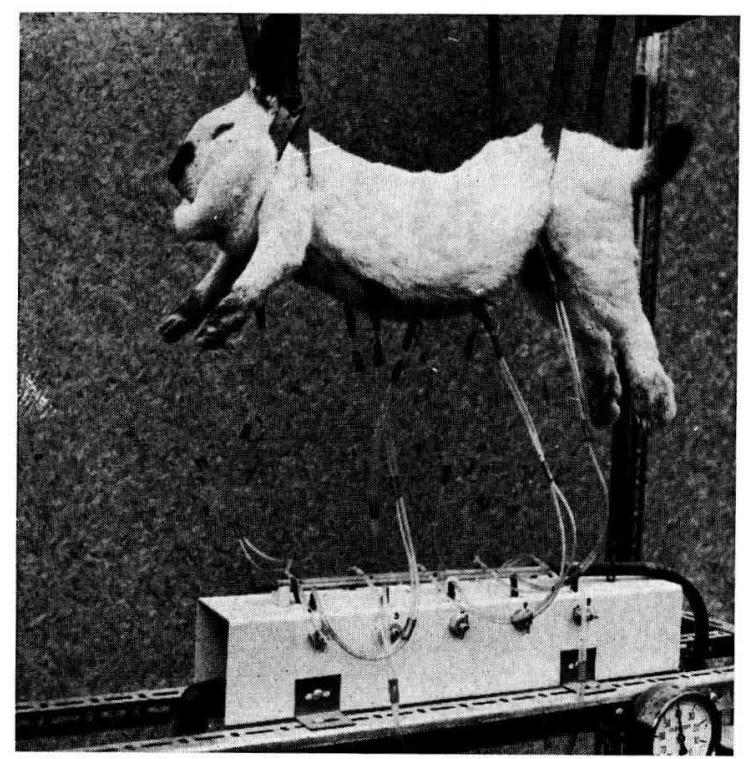

FIG. I. - Machine à traire en fonctionnement

Le générateur de pulsation est identique à celui utilisé pour les vaches laitières. La cadence correspond dans notre cas à 45 pulsations par minute. Le vide alternatif est distribué simultanément à l'ensemble des gobelets trayeurs grâce à un tube rigide de $\mathrm{I} \mathrm{cm}$ de diamètre comportant Io sorties opposées 2 à 2 et réparties sur la longueur d'une lapine environ. Un tuyau plastique souple le relie au gobelet trayeur proprement dit. La traite est réalisée avec un vide de $50 \mathrm{~cm}$ de mercure au maximum.

Le dispositif de réception du lait est un flacon de $500 \mathrm{~cm}^{3}$ unique pour l'ensemble des trayons, relié d'une part à la source de vide et d'autre part aux gobelets trayeurs. Cette dernière liaison est réalisée par un ensemble de robinetterie qui permet d'utiliser un nombre quelconque des ro gobelets trayeurs présents. L'ensemble des tuyauteries est réalisé de manière à ce qu’en position de travail, le lait puisse s'écouler 
naturellement du gobelet trayeur au flacon de réception sans qu'il puisse y avoir d'accumulation dans une partie aveugle ou en forme de siphon.

Le gobelet trayeur (fig. 2) est constitué par un tube de cuivre dans lequel passe librement une feuille anglaise. Celle-ci est rabattue à la partie supérieure, au contact du téton de la lapine. Dans sa partie inférieure elle est emboîtée sur le tuyau plastique (1) ; une gaine constituée par de la feuille anglaise d'un diamètre légèrement

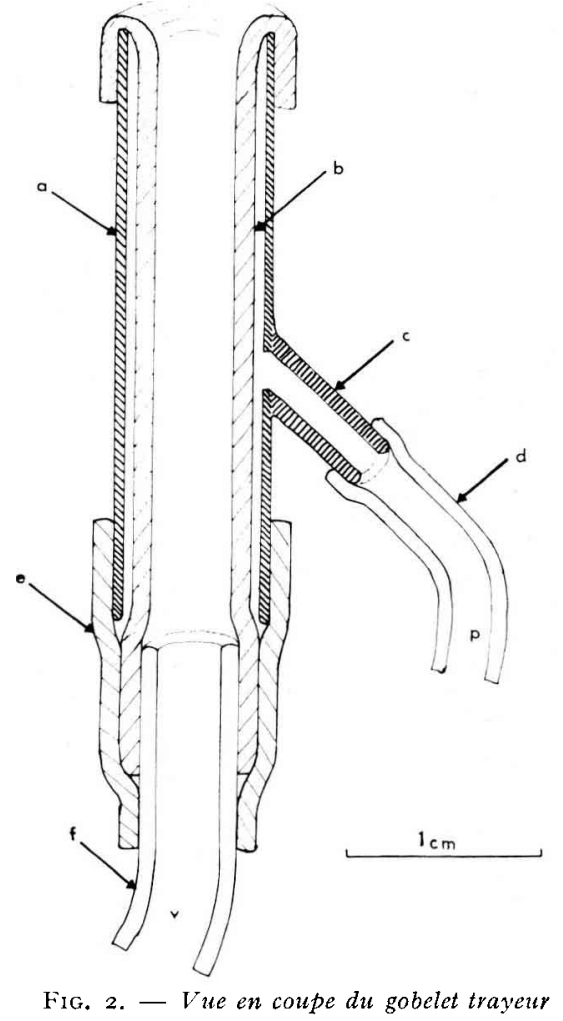

Les chiffres indiqués après les désignations correspondent dans l'ordre au diamètre extérieur puis intérieur en mm. $a$ : corps du gobelet, tube de cuivre 8-7. $b$ : feuille anglaise 6-4. $c$ : arrivée pulsation tube cuivre $3-\mathrm{I}, 5 . d:$ tube plastique $3,5-2 . e:$ gaine feuille anglaise 7-5. $f:$ tube d'évacuation du lait, en plastique $5-3,4 . p:$ pulsation. $v$ : vide continu.

supérieur à la précédente assure l'étanchéité. L'autre extrémité du tuyau plastique de $30 \mathrm{~cm}$ de long, est branchée sur l'un des robinets relié au dispositif d'aspiration et de collecte du lait. Dans la partie inférieure du tube de cuivre, un orifice est percé, un petit tube de cuivre y est adapté, puis soudé. Il est lui-même relié à la distribution de vide alternatif par un tuyau plastique de diamètre plus faible que le précédent $\left(^{2}\right)$.

L'utilisation de tuyaux plastiques pour le transport du lait avant le système de collecte général permet une surveillance aisée de l'écoulement de chacun des trayons de la lapine. La réception dans un flacon de verre autorise le contrôle des quantités de lait obtenues.

(1) Tube de manchon Cie Technicon.

(2) Tube de liaison Cie Technicon. 


\section{B. - Le système de contention}

La mise en place des gobelets trayeurs nécessite le libre accès atux trayons situés sur la partie ventrale de la lapine. Après avoir essayé plusieurs systèmes dont une boîte à contention à fond grillagé et un hamac laissant passer les membres, nous avons adopté un système de harnais.

La lapine est suspendue au-dessus de la machine à traire par un ensemble de courroies de cuir de $2 \mathrm{~cm}$ de large venant prendre la femelle au niveau des membres (fig. 3). Les pattes antérieures sont passées dans deux anneaux de cuir reliés entre eux à la partie inférieure par une mince bande de cuir. Deux courroies permettent d'accrocher les anneaux au châssis de la machine à traire.

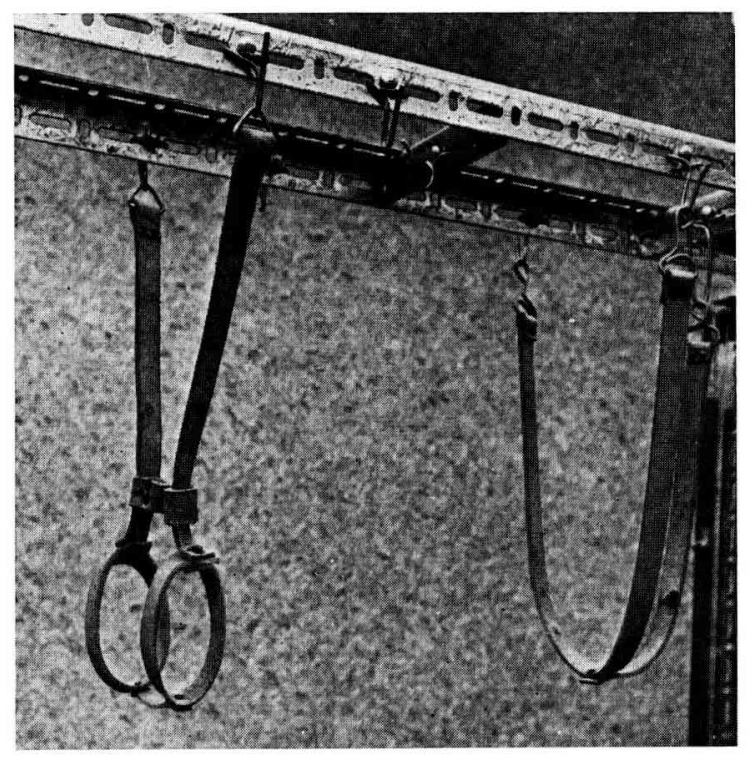

FIG. 3. - Système de harnais destiné à soutenir la lapine

Une sangle passe sous le ventre de la femelle juste en avant des cuisses. Au point le plus bas une autre sangle est fixée; elle est passée entre les 2 cuisses et remonte sous la queue de l'animal. Mises à plat, ces 2 sangles ont la forme d'un T dont la barre passerait sous le ventre et le jambage entre les cuisses et sur la queue.

Ce système de harnais permet un libre accès à toutes les tétines y compris les tétines inguinales. Cependant, il est parfois nécessaire de dégager à la main une ou deux tétines pectorales qui se trouvent prises sous la bande de cuir reliant les 2 anneaux antérieurs. Cet inconvénient est réduit si cette bande est plus étroite $(0,5 \mathrm{~cm}$ de large). 


\section{II. - TECHNIQUE DE TRATTE}

L'utilisation de cette machine à traire, comme la traite manuelle, nécessite une injection d'ocytocine. Nous pratiquons une injection intra-veineuse de I à $2 \mathrm{~cm}^{3}$ d'une solution d'extrait post-hypophysaire titrant I UI $/ \mathrm{cm}^{3}$. Cette injection est réalisée dans la veine marginale de l'oreille avant la mise en place de la lapine sur la machine à traire. L'animal est alors harnaché rapidement et mis en place pour la traite (fig I).

Après un temps de latence de 3 à 4 minutes suivant l'injection, variable d'ailleurs avec les animaux, le lait commence à couler par l'ensemble des trayons. Une soupape permet de régler la dépression que nous utilisons à un maximum de $50 \mathrm{~cm}$ de mercure, mais il est possible de traire à partir de $30 \mathrm{~cm}$ de mercure. Une telle variation du niveau de vide est expliquée par le manque d'étanchéité au niveau des tétines par suite de la présence de poils.

Le volume à traire est prédéterminé par un trait de jauge sur le flacon de réception. Cette technique permet de réaliser des prélèvements de lait correspondant exactement aux quantités moyennes consommées les 2 jouts précédents par les lapereaux. Il nous a été donné de constater qu'une portée de 7-8 lapereaux, par exemple, n'obtient en général pas la totalité du lait présent dans la mamelle au moment de la tétée, mais une fraction n'en représentant que 75 à $95 \mathrm{p}$. Ioo, l'extraction naturelle par une portée d'effectif moyen est donc moins efficace que celle pratiquée grâce à la machine, appliquée au même rythme de I fois par 24 heures. La durée de traite est de 5 à 6 minutes soit un peu plus que le temps de tétée normal (CRoss, I952).

\section{REMARQUES}

Pour un bon fonctionnement des gobelets trayeurs, il est parfois nécessaire de leur appliquer une légère traction qui facilite l'écoulement du lait de la mamelle.

Pour une traite bilatérale la présence de 2 trayeurs est nécessaire. Si 1'on veut obtenir une vidange complète de la totalité des mamelles, il est utile de prévoir 4 trayeurs de manière à réduire au minimum les prises d'air au niveau des tétines et à assurer la légère traction mentionnée ci-dessus.

Nous n'avons pas cherché à obtenir séparément le lait de chaque trayon ; sa connaissance ne serait que d'un intérêt purement théorique compte tentu des changements de téton opérés par les lapereaux d'une tétée à l'autre, voire au cours d'une même tétée.

\section{CONCLUSIONS}

La machine décrite ci-dessus permet de réaliser des prélèvements réellement représentatifs du lait consommé par les lapereaux. L'injection nécessaire d'ocytocine peut être considérée encore comme un inconvénient, mais nous ne pensons pas qu'elle puisse avoir une incidence sur la composition du lait prélevé dans les 6 à 8 minutes 
suivant son administration. Les pièces nécessaires à la fabrication de cette machine sont d'une fourniture ou d'une fabrication aisée. Son emploi n'offrant pas de difficulté majeure, elle devrait permettre d'améliorer notre connaissance du phénomène " lactation " chez la Lapine.

\section{SUMMARY}

\section{DESCRIPTION OF A MILKING MACHINE FOR RABBITS}

When rabbits are hand milked, even after injection of oxytocin, only a small part of the milk present in the mammary gland may be obtained. The milking machine for rabbits described in this paper was constructed on the same principle as that of the milking machine for cows. The rabbit is suspended in a harness over the collecting system, which includes Io teatcups and one only milk holder. The pulsation rate is 45 minutes and the vacuum varies between 30 and $50 \mathrm{~cm}$ mercury, according to tightness. Each teatcup is composed of a metal shell and a rubber liner. A simple rubber sheath ensures tight connection with the milk tubes. When using this machine, one may collect an amount of milk equivalent to or higher than that ingested by the young rabbits at one suckling.

\section{REMERCIEMENTS}

Nous tenons à remercier la Société “ Lorraine " pour la fourniture gracieuse du pulsateur de la machine à traire.

\section{RÉFÉRENCES BIBLIOGRAPHIQUES}

BergmanN A. J., TuRner C. W, 1937. The composition of rabbit milk stimulated by the lactogenic hormone. J. Biol. Chem., 120, 2 I-27.

Contes M. E., Gregory M. E., Thompson S. Y., 1964, Composition of rabbit's milk. Brit., J. Nutr., 18, 583-586.

Cowre A. T., I969. Variations of the yeld and composition of the milk during lactation in the rabbit and the galactopoietic effect of prolactin. J. Endocr., 44, 437-450.

Cross B. A., r952. Nursing behavior and the milk ejection reflex in rabbits. J. Endocr., 8, Proc. XIII-XIV.

Davies J.S., Widdowson E. M., Mac Rance R. A., 1964. The intake of milk and the retention of its constituents while the newborn rabbit doubles its weight. Brit. J. Nutr., 18, 385-392.

Neumeister H., Krause B., 1956. Zum Nährstoffgehalt der Kaninchenmilch. Arch. Geflïgelz. Kleintierk., 5, 199-203.

Nixon D. A., 1953. The free inositol content of milk. J. Physiol., 120, 27 p. 